\title{
Influence du risque suicidaire et du désespoir sur l'amplitude de l'onde P300 dans la dépression majeure
}

\author{
I Urcelay-Zaldua ${ }^{1}, M$ Hansenne $^{1-2}, \mathrm{M}^{\text {Ansseau }}{ }^{2}$ \\ 'Unité de psychiatrie, CHU André-Vésale, 706, route de Gozée, B-6110, Montigny-le-Tilleul; \\ ${ }^{2}$ unité de psychiatrie, CHU du Sart-Tilman (B35), B-4000 Liège, Belgique
}

(Reçu le 10 février 1995; accepté le 1er juin 1995)

\begin{abstract}
Résumé - L'onde P300 constitue un outil intéressant pour l'étude du traitement de l'information. Dans la dépression, plusieurs études ont mis en évidence une diminution de l'amplitude de la P300 et une augmentation de sa latence. Néanmoins, la signification fonctionnclle des résultats reste imprécise. Dans cette étude nous avons évalué l'influence du risque suicidaire et du désespoir sur l'amplitude de la P300 chez 40 patients déprimés. Les résultats montrent une relation négative entre l'amplitude de la P300 et le risque suicidaire $(\mathrm{r}=-0,68, p<0,001)$ ainsi qu'avec le désespoir $(\mathrm{r}=-0,76, p<0,001)$. D'un point de vue clinique, ces résultats indiquent que l'amplitude de la P300 pourrait être considérée comme un index psychophysiologique du risque suicidaire dans la dépression majeure.
\end{abstract}

P300 / dépression / risque suicidaire / désespoir

Summary - Influence of suicidal risk and hopelessness on P300 amplitude in major depressive disorder. $P 300$ amplitude represenis u useful tool to assess information processing in normal and psychopathological subjects. In depressive disorders, many studies have shown a decrease of P300 amplitude and an increase of its latency. However, functional significance of the P300 modifications remain unclear. The aim of the study was to assess the influence of suicidal risk and hopelessness on P300 amplitude among 40 depressive inpatients. The results showed significant relationships between P300 amplitude and suicidal risk $(r=-0.68, p<0.001)$ and with hopelessness $(r=-0.76, p<0.001)$. From a clinical point of view, $P 300$ amplitude should be considered as a psychophysiological index of suicidal risk in major depressive disorder.

P300 / depressive disorders / suicidal risk / hopelessness

\section{INTRODUCTION}

Les potentiels évoqués endogènes, et plus particulièrement l'onde $\mathrm{P} 300$, constituent une approche intéressante pour l'étude du traitement de l'information chez le sujet

Correspondance et tirés à part: M Hansenne, unité de psychiatrie, CHU du Sart-Tilman (B35), B-4000 Liège, Belgique. 
normal et en psychopathologie (Donchin et al, 1986; Charles et Hansenne, 1992). L'amplitude de l'onde P300 est influencée par de nombreuses variables, comme la probabilité du stimulus, sa signification, ainsi que la quantité d'information qu'il fournit (Johnson, 1986). D'un point de vue théorique, l'onde P300 reflèterait le processus d'adaptation entre la mémoire de travail et l'environnement «context updating» (Donchin et Coles, 1988), ainsi que la vitesse du traitement de l'information (Kutas et al, 1977).

Levit et al (1973) ont été les premiers à montrer que l'amplitude de l'onde P300 était réduite dans un groupe de déprimés, et se situait entre celles des sujets normaux et des patients schizophrènes. Par la suite, différentes études ont confirmé la diminution de l'amplitude de l'onde P300 dans la dépression majeure, associée à une augmentation du temps de réaction (Roth et al, 1986; Pfferferbaum et al, 1984), alors que d'autres études n'ont pas confirmé ces résultats (Gordon et al, 1986; Patterson et $a l, 1988$ ). Ainsi, la signification fonctionnelle de la diminution de l'onde P300 reste imprécise.

Plus récemment, plusieurs études ont séparé différents groupes de dépression en fonction d'une variable clinique. Santosh et al (1994) ont mis en évidence une diminution plus importante de l'amplitude de l'onde P300 chez des patients déprimés avec des caractéristiques psychotiques par rapport à des patients déprimés sans caractéristiques psychotiques. Pierson et al (1991) ont montré une différence de l'amplitude de la P300 entre un groupe de patients déprimés caractérisé par un émoussement des affects et un autre groupe caractérisé par une impulsivité.

Par ailleurs, dans une étude préliminaire, nous avons trouvé une amplitude de la P300 plus faible chez des patients déprimés qui venaient de réaliser une tentative de suicide par rapport à des patients déprimés sans antécédents suicidaires (Hansenne et $a l, 1994)$. De plus, il existait une relation entre le risque suicidaire et l'amplitude de l'onde P300.

Le but de notre travail est d'étudier l'influence de variables cliniques sur l'amplitude de l'onde P300 dans la dépression majeure afin de contribuer à une meilleure connaissance de la valeur fonctionnelle de cette onde. Plus spécifiquement, nous voulons confirmer l'influence du risque suicidaire sur l'amplitude de la P300 dans un plus grand échantillon, et de tester le rôle de la variable «désespoir».

\section{MÉTHODE}

Quarante patients déprimés selon les critères diagnostiques du DSM-III-R (APA, 1987) ont participé à l'étude (hôpital universitaire André-Vésale, unité de psychiatrie, Montignyle-Tilleul, Belgique). L'échantillon comprenait 20 hommes (âge moyen 35,1 ans $\pm 12,4$ ) et 20 femmes (âge moyen 38,5 ans $\pm 15,3$ ). Quatorze (sept hommes et sept femmes) des patients avaient été inclus dans l'étude préliminaire (Hansenne et al, 1994). Les patients ne présentaient pas de maladies organiques mises en évidence par un examen clinique exhaustif, une biologie de routine, ECG, EEG et une radio du thorax. Les patients avaient un score supérieur à 18 à l'échelle de dépression de Hamilton à 17 items. L'échelle du risque suicidaire (Plutchick et al, 1989) a été remplié par la totalité des patients (dont 14 faisaient partie de l'étude précédente), et l'échelle du désespoir (Beck et al, 1985) par les 26 nouveaux patients.

L'enregistrement psychophysiologique a été réalisé après une période de sevrage médicamenteux d'au moins 1 semaine. Les patients étaient assis dans une pièce insonorisée. Pour le paradigme P300, une série de 100 stimuli auditifs était présentée avec un intervalle inter stimuli aléatoire ( 2 à 4 secondes). Les stimulations fréquentes étaient délivrées avec une pro- 


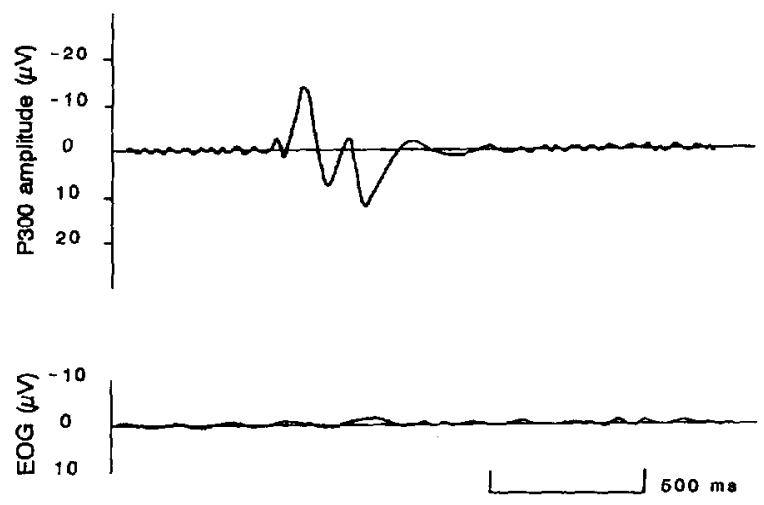

Fig 1. Moyenne totale des ondes P300 obtenues chez nos 40 patients déprimés.

babilité de $85 \%$ et une fréquence de $2000 \mathrm{~Hz}(75 \mathrm{db}, 50 \mathrm{~ms})$, et les stimulations rares avec une probabilité de $15 \%$ et une fréquence de $1000 \mathrm{~Hz}(75 \mathrm{db}, 50 \mathrm{~ms})$ afin d'obtenir un total de 16 tracés non artéfactés. Il était demandé aux patients d'appuyer sur un bouton poussoir à chaque stimulation rare. L'amplitude de l'onde P300 était mesurée comme la différence de potentiel entre la ligne de base ( 1 seconde), et l'amplitude maximale comprise entre 250 et $650 \mathrm{~ms}$.

L'enregistrement était réalisé à l'aide d'électrodes $\mathrm{Ag} / \mathrm{AgCl}$ attachées avec du collodion au vertex $(\mathrm{Cz})$, avec le lobe de l'oreille gauche comme référence, et le milieu du front droit comme terre. La peau était préparée avec une pâte abrasive et de l'acétone afin de maintenir les impédances en dessous de 3 KOhms. L'électro-oculogramme (EOG) était enregistré à l'aide d'une électrode placée en dessous de l'œil gauche. Un EOG supérieur à $50 \mu \mathrm{V}$ entraînait un rejet automatique. Seuls les tracés non artéfactés et sans erreur de discrimination ont été moyennés. Le gain de l'amplificateur était fixé à 10,000 , avec une bande passante comprise entre 0,05 et $50 \mathrm{~Hz}$. Les signaux étaient digitalisés à 200 points par seconde.

\section{RÉSULTATS}

La figure 1 présente la moyenne des tracés électrophysiologiques obtenus chez nos 40 patients déprimés. L'amplitude moyenne de l'onde P300 était de 11,63 $\mu \mathrm{V}$ $( \pm 7,7)$, et la latence de $335,4 \mathrm{~ms}( \pm 23,2)$. Le temps de réaction était de $485 \mathrm{~ms}$ $( \pm 158)$. Parmi les 40 patients, le score moyen à l'échelle du risque suicidaire était de 14,12 $( \pm 4,35)$. Le score moyen de l'échelle de désespoir chez 26 patients était de $8,95( \pm 3,74)$.

Les résultats ont mis en évidence une corrélation négative entre l'amplitude de la P300 et l'échelle du risque suicidaire $(r=-0,68, p<0,001)$ (fig 2a) ainsi qu'avec l'échelle de désespoir ( $\mathrm{r}=-0,76, p<0,001)$ (fig $2 \mathrm{~b}$ ), mais pas avec l'échelle de dépression de Hamilton $(\mathrm{r}=0,10, p=0,52)$. L'échelle du risque suicidaire était fortement corrélée avec l'échelle de désespoir $(p=0,82, p<0,001)$.

En revanche, le temps de réaction et la latence de l'onde $\mathrm{P} 300$ ne présentaient aucune relation avec les échelles d'évaluations utilisées dans notre étude. De plus, l'àge des patients n'était pas corrélé avec les données électrophysiologiques et psychométriques. 


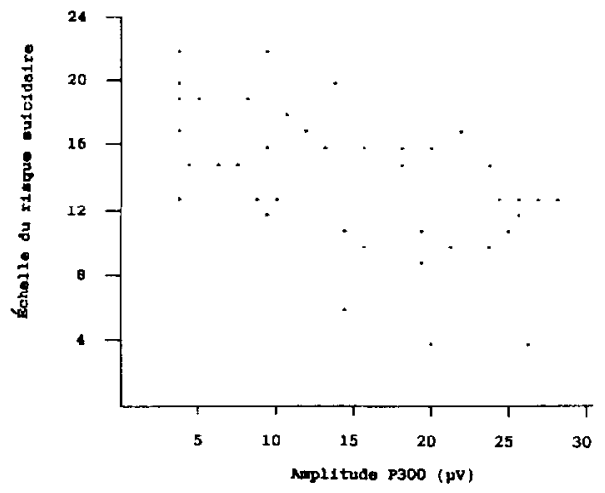

Fig 2a. Distribution de la relation entre le risque suicidaire et l'amplitude de l'onde P300 dans notre échantillon de 40 patients déprimés.

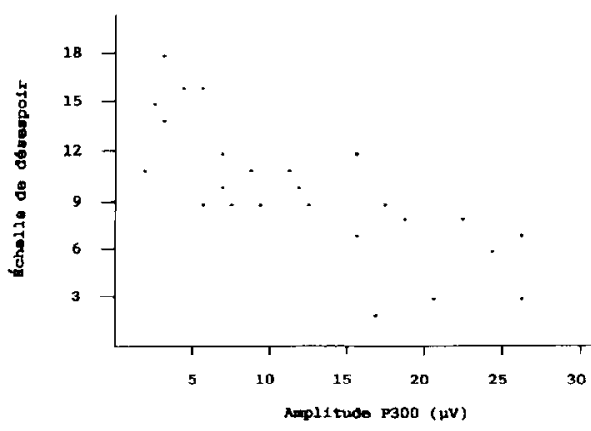

Fig 2 b. Distribution de la relation entre le désespoir et l'amplitude de l'onde P300 dans notre échantillon de 26 patients déprimés.

\section{DISCUSSION}

Notre étude a mis en évidence une relation négative entre l'amplitude de la P300 et le risque suicidaire, confirmant ainsi notre étude préliminaire (Hansenne et al, 1994). De plus, une corrélation négative a été observée entre l'amplitude de la P300 et l'échelle de désespoir. En revanche, nous n'avons pas trouvé de relation entre le temps de réaction et la latence de l'onde P300 et les échelles psychométriques, alors que certaines études ont mis en évidence des différences pour ces paramètres entre des populations cliniques et des sujets normaux (Thier et al, 1986). La simplicité du protocole utilisé dans notre étude peut expliquer cette contradiction. Par ailleurs, notre étude présente d'importantes limitations. Tout d'abord, l'absence de groupe contrôle ne permet pas de déterminer si les relations décrites sont spécifiques de la dépression majeure. Ensuite, l'utilisation d'un seul canal d'enregistrement limite la généralisation des résultats.

D'un point de vue théorique, l'amplitude de l'onde P300 est influencée par de nombreuses variables psychologiques chez les sujets normaux, et leur interaction a été modélisée par Johnson (1986). Dans notre étude, le risque suicidaire et le désespoir influencent significativement l'amplitude de l'onde P300 dans la dépression majeure. Néanmoins, comme les deux échelles cliniques sont fortement corrélées, elles ne peuvent pas être considérées comme deux entités différentes. D'ailleurs, le désespoir semble constituer une variable capitale pour la prédiction du risque suicidaire (Beck, 1986).

Rockstroh et al (1982) ont proposé un modèle intégré du fonctionnement du système nerveux central combinant deux concepts. Il s'agit de la potentialité cérébrale, représentée par une déflexion négative du cortex, et de la performance cérébrale, représentée par une déflexion positive. Plus récemment, ces auteurs ont développé et nuancé leur interprétation des potentiels lents (Birbaumer et al, 1990). Ils considèrent que les potentiels lents reflètent la modulation de l'excitabilité corticale par rapport à un seuil déterminé. Les déflexions négatives sont associées à une augmentation de l'excitabilité corticale et s'observent lors de tâches d'anticipations, alors que les déflexions positives (P300) sont associées à une diminution transitoire de l'excitabilité corticale nécessaire pour accomplir une opération mentale comme l'accès à 
la mémoire de travail ou le stockage d'informations. En expliquant nos résultats en faisant référence à ce modèle, nous pouvons émettre l'hypothèse que les deux variables cliniques étudiées influencent négativement l'excitabilité corticale et les performances. En effet, les patients déprimés avec une idéation suicidaire et un important désespoir possèdent moins de ressources par rapport à l'environnement, dans la mesure où ils n'attendent que des effets négatifs de leurs comportements. Ainsi, l'augmentation de l'excitabilité corticale n'est pas suffisante pour produire une performance adéquate.

D'un point de vue pharmacologique, différentes études montrent que la fonction dopaminergique est altérée chez des patients déprimés avec un risque suicidaire important (Roy et al, 1992; Agren, 1980; Pitchot et al, 1992). Par ailleurs, l'amplitude de la P300 a été mise en relation avec, entre autres, le système dopaminergique (Timsit-Berthier, 1991 ; Callaway, 1991; Hansenne et al, 1995). Ainsi, l'association entre le risque suicidaire et l'amplitude de la P300 rapportée dans cette étude pourrait refléter le dysfonctionnement dopaminergique mis en évidence dans les comportements suicidaires.

\section{RÉFÉRENCES}

Agren H. Symptom patterns in unipolar and bipolar depression correlating with monoamine metabolites in the cerebrospinal fluid: II, Suicide. Psychiatry Res 1980;3:225-36

American Psychiatric Association. Diagnostic and Statistical Manual of Mental Disorders (ed 3 revised). Washington, American Psychiatric Association, 1987

Beck AT, Steer RA, Kovacs M, Garisson M. Hopelessness and eventual suicide: a 10-year prospective study of patients hospitalized with suicidal ideations. Am J Psychiatry 1986; 142:559-63

Beck AT. Hopelessness as a predictor of eventual suicide. In: Mann JJ, Stanley M, eds. Psychobiology of suicidal behavior. Ann NYAcad Sci 1985;487:90-6

Birbaumer N, Elbert T, Canavan AGM, Rockstroh B. Slows potentials of the cerebral cortex and behavior. Physiol Rev 1990;70:1-41

Callaway E, Discussion: towards a pharmacology of information processing. In: Brunia CHM, Mulder G, Verbaten MN, eds. Event-Related Brain Research, EEG suppl 42. Amsterdam: Elsevier, 1991:173-6

Charles G, Hansenne M. Le potentiel lent P300. Intérêts cliniques dans trois pathologies et neurobiologie: une revue. L'encéphale 1992; 18:225-36

Donchin E, Karis D, Bashore TR, Coles MGH, Gratton G. Cognitive psychophysiology and human information processing psychophysiology. In: Cole MGH, Donchin E, Porges SW, eds. Systems, processes and applications. Amsterdam: Elsevier, 1986:244-67

Donchin $\mathrm{E}$, Coles $\mathrm{M}$. Is the $\mathbf{P} 300$ component a manifestation of context updating? Behav Brain Sci 1988; 1 1:357-74

Gordon E, Kraiuhin C, Harris A, Meares R, Howson $A$. The differential diagnosis of de- mentia using P300 latency. Biol Psychiatry 1986;21:1123-32

Hansenne M, Pitchot W, Gonzalez-Moreno A Gonzalez-Torrecilas J, Mirel J, Ansseau M. Psychophysiological correlates of suicidal behavior in depression: preliminary results. Neuropsychobiology 1994;30:1-3

Hansenne M, Pitchot W, Gonzalez-Moreno A. Papart P, Timsit-Berthier $\mathbf{M}$, Ansseau $\mathbf{M}$. Catecholaminergic functions and P300 amplitude in depressed patients. Electroencephalogr Clin Neurophysiol 1995;96:1946

Johnson R. A triarchic model of P300 amplitude. Psychophysiology 1986;23:367-84

Kutas M, Carthy G, Donchin E. Augmenting mental chronometry: the P300 as a measure of stimulus evaluation time. Science 1977; 197:792-5

Levit RA, Sutton S, Zubin J. Evoked-potential correlates of information processing in psychiatric patients. Psychol Med 1973;3:487-94

Patterson JV, Mikaleski HJ, Starr A. Latency variability of the components of auditory event related potentials to infrequent stimuli in aging, Alzheimer-type dementia and depression. Electroencephalogr Clin Neurophysiol 1988;71:450-60

Pearson A, Partiot A, Ammar S, Dodin V, Loas G, Jouvent R, Renault B. ERP differences between anxious-impulsive and blunded-affect depressive inpatients. In: Ansseau M, von Krenckell R, Franck G, eds. Biological markers of depression: State of the art. Amsterdam: Elsevier, 1991:121-9

Pfefferbaum A, Wenegrt BG, Ford JM. Clinical applications of the $\mathrm{P} 300$ components of event related potentials. II. Dementia, depression and schizophrenia. Electroencephalogr Clin Neurophysiol 1984;59:104-24

Plutchick R, van Praag HM, Conte HR, Pilard S. 
Correlates of suicide and violence risk 1: The suicide risk measure. Compr Psychiatr 1989; 30:296-302

Pitchot W, Hansenne M, Gonzalez Moreno A Arsseau M. Suicidal behavior and growth hormone response to apomorphine test. Biol Psychiatry 1992;31:1213-9

Rockstroh B, Elbert T, Lutzenberger W, Birbaumer N. Slow brain potentials and behavior. Urban and Schwarzenberg, 1982

Roth WT, Duncan CC, Pfefferbaum A, TimsitBerthier M. Applications of cognitive ERPs in psychiatric patients. In: McCallum WC. Zappoli R, Denoth F, eds. Cerebral psychophysiology: Study in Event-Related Potentials, EEG suppl 38. Amsterdam: Elsevier, 1986 : 419-38
Roy A, Karoum F, Pollack S. Marked reduction in indexes of dopamine metabolism among patients with depression who attempt suicide. Arch Gen Psychiatry 1992:9:447-50

Santosh PJ, Malhotra S, Raghunathan M, Mehra YN. A study of P300 in melancholic depression. Correlation with psychotic features. Biol Psychiatry 1994;35:474-9

Timsit-Berthier M. Contingent negative variation (CNV) in psychopharmacology. In: Brunia CHM, Mulder G, Verbaten MN, eds. EventRelated Brain Research, EEG suppl 42. Amsterdam: Elsevier, 1991:142-52

Thier P, Axmann D, Giedke H. Slow brain potentials and psychomotor retardation in depression. Electroencephalogr Clin Neurophysiol 1986;63:570-81 